\section{For patient confidence in your practice}

When you welcome patients back to the practice, you want them to feel safe.

Reinforce your infection control protocols with the Steri-7 Xtra range from Initial Medical, a high-quality cleaning and disinfectant solution for surfaces and hands.

Steri-7 Xtra High Level Surface Disinfectant Cleaner is available as a concentrated solution, a ready to use spray, and a surface wipe. The Steri-7 Xtra Personal Care Range includes a hypoallergenic surgical level hand rub and wash, both of which are effective and kind to skin.

Steri-7 Xtra deactivates up to $99.999 \%$ of a broad range of pathogens within seconds of contact and has been tested against feline coronavirus: a surrogate for coronavirus. Steri-7 Xtra surface sanitisers utilise specially formulated micro emulsion technology that applies an active barrier which can persist and kills for hours afterward. Initial Medical's supplier's laboratory testing showed the barrier killing microbes (including pathogens) 72 hours after application, so long as the barrier is not wiped away.

With Steri-7 Xtra, your patients can be confident about how protected they are.

You can buy the Steri-7 Xtra range online: https://www.initial.co.uk/ infection-control/surface-hygiene/.

For further information visit www. initial.co.uk/medical or tel: 08708504045 .

\title{
Optimum comfort, efficient endodontics
}

For canal preparation, the HyFlex EDM file sequence from COLTENE is suitable for everyday endodontics and complex situations.

Robust yet flexible, the files allow for efficient treatment delivery. HyFlex EDM files can be pre-bent to follow the anatomy of the canal, reducing the risk of ledging, perforation and transportation.

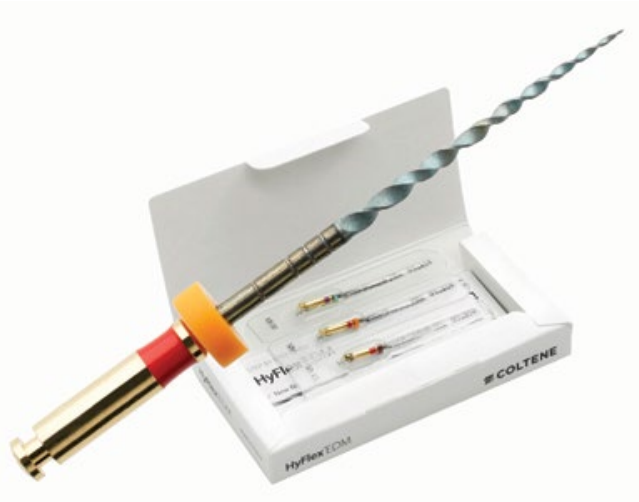

The options that HyFlex EDM files offer mean that only one or two files may be needed; it's a cost-effective system. All your patients, including those who are elderly, will be treated comfortably with their tooth restored to health.

Go to the website for a full list of benefits of the HyFlex ${ }^{\text {ma }}$ EDM file system.

To find out more visit www.coltene. com, email info.uk@coltene.com or call 01444235486.

\section{Dentists on demand}

Waterpik is delighted to have partnered with the Tooth Fairy App, helping to connect patients with dentists during these difficult times.

The Tooth Fairy App enables patients to speak to qualified and experienced dentists for virtual consultations using video calling facilities. It was designed by dentists to provide a safe, cost-effective and convenient way for people to access reliable dental advice and information.

For clinicians, it provides a new and innovative way of reaching out to patients, delivering the guidance they need and marketing to a brand-new audience. The app has already won App of the Year 2019 at the Dental Industry Awards, being highly commended in the High Technology Launch category of the same year.

Having supported the dental care profession for many years, Waterpik aims to help even more patients improve their oral health through its partnership with the Tooth Fairy App. For more information, visit www.toothfairydentist. co.uk or download the app from the App Store.

For more information on the Waterpik Water Flosser visit www.waterpik.co.uk. Waterpik products are available from Amazon and in store or online at Asda, Boots and Superdrug.

\section{Restart and accelerate your implant business}

BioHorizons Camlog has launched the Accelerate Bounceback initiative providing a wide range of solutions and tools to the dental industry to help restart businesses and accelerate out of the COVID-19 crisis to the new normal of implant dentistry.

Through its extensive partner network and business development tools,

BioHorizons Camlog are able to provide dental professionals with a wide array of solutions ranging from checklists, to best practice videos from dentists, product offers tailored to customers' needs, business and clinical webinars, patient 'Safety First' campaign collateral for all practices to use themselves and much more.

The BioHorizons Camlog team is fully operational and is available to help restart businesses and accelerate successfully out of the COVID-19 crisis to the new normal of implant dentistry: be that through implant bundles providing FOC air purification solutions or a 12 month teledentistry licence, increased FOC biologics with no increased minimum spend, a patient education campaign showing that practices are implementing procedures to keep patients safe, online education or simply to lend an ear when dental professionals plan the next business procedures.

Whatever is needed, contact

BioHorizons Camlog today.

For further information on the Accelerate Bounceback initiative visit www.acceleratebounceback.com or contact BioHorizons Camlog on +44 (0) 1344 752560 orinfouk@biohorizons.com. 